\title{
ADDITIVE LASER BARCODE PRINTING ON HIGH REFLECTIVE STAINLESS STEEL
}

\author{
Mihail Stoyanov Mihalev ${ }^{a, *}$, Chavdar Momchilov Hardalov ${ }^{b}$, \\ Christo Georgiev Christov ${ }^{b}$, Monika Rinke $^{c}$, Harald Leiste ${ }^{c}$, \\ JOHANNES SCHNEIDE ${ }^{d}$
}

${ }^{a}$ Technical University of Sofia, Faculty of Mechanical Engineering, Department of Precision Engineering and Measurement Instruments, $8 \mathrm{Kl}$. Ohridski blvd, 1000 Sofia, Bulgaria

${ }^{b}$ Technical University of Sofia, Department of Applied Physics, 8 Kl. Ohridski blvd, 1000 Sofia, Bulgaria

${ }^{c}$ Karlsruhe Institute of Technology, Institute of Applied Materials - Applied Materials Physics, Hermann-von-Helmholtz-Platz 1, 76344 Eggenstein-Leopoldshafen, Germany

${ }^{d}$ Karlsruhe Institute of Technology, Institute of Applied Materials - Computational Materials Science, Hermann-von-Helmholtz-Platz 1, 76344 Eggenstein-Leopoldshafen, Germany

* corresponding author: mmihalev@tu-sofia.bg

ABstract. In this paper, the process of an additive laser marking on stainless steel parts for barcode printing is presented. It is based on the use of one transition metal oxide chemically-well bonded to the stainless steel substrate, without a usage of any additional materials and cleaning substances.

The resulting additive coatings, produced from initial $\mathrm{MoO}_{3}$ powder by irradiation with a laser beam, reveal strong adhesion, high hardness, long durability and high optical contrast, which make the process suitable for barcode printing on materials such as high reflective stainless steel, which was always a challenge for the classical laser marking technologies.

The obtained bar patterns are in a compliance with the requirements of the existing standards.

KEYWORDS: Additive laser marking, direct part marking, $\mathrm{MoO}_{3}$, transition metal oxides, chemical bonding.

\section{INTRODUCTION}

Nowadays, the product information becomes more and more important due to the globalization of the world industry. This kind of production requires a consistent system for identification and classification of the products.

Since 1952, the bar and 2D codes are established as a standard tool for identifying and classification of the quality in the industrial part production, part assembling, logistics and traceability. The codes carry information about the origin, the history of the production, assembling and transportation needed by the companies for improvement of their quality procedures and help avoiding errors before the products are shipped. To meet these challenges, the barcodes were standardized in ISO/IES 15415 [1].

NASA also developed a standard for matrix identification of their numerous details aiming at reliable synchronization during the production and assembling time [2].

This approach is followed by many other federal agencies and departments, such as the Department of Defense as well as many companies in USA and EU.

The marking process plays a key role for the reliable recognition of the barcode symbols and identification of the details. From a technological point of view, the marking coating is expected to have a high optical contrast in a broad spectral range as well as a good adhesion to the substrate and must be durable as well.

One of the most versatile techniques is the laser marking. However, the inscription of metal parts, especially of highly reflective stainless steel with standard material subtractive methods like laser ablation is a challenge for this approach due to the low contrast, change of the substrate material properties during the laser treatment and emerging of habitats of micro-flora and fauna, which makes it unacceptable for marking of medical instruments, for example. To overcome most of the mentioned disadvantages, we present an additive marking process, which needs only one transition metal oxide for the preparation of a dark coloured coating, chemically-well bonded to the stainless steel substrate, without any additional materials and cleaning substances [3, 4]. Henceforth, it will be referred to as "Additive Laser Marking" (ALM).

The aim of the present publication is to reveal the feasibility of the ALM to be used for a specific industrial application, namely marking barcodes on highly reflective stainless steel and the compliance of the obtained barcodes with the standards. 


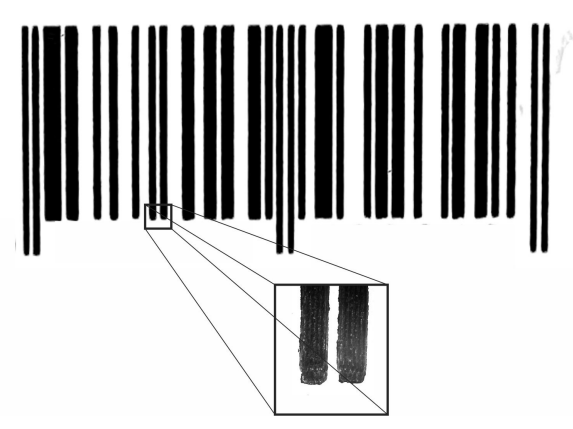

Figure 1. Digital photo of a real ALM barcode with a microscopic image of selected black bars (square below the barcode).

\section{EXPERIMENTAL}

\subsection{TeChNology of ADDitive LASER DIRECT PART MARKING}

A plate of mirror finished stainless steel Grade 304 is chosen as a substrate and $\mathrm{MoO}_{3}$ powder as a typical transition metal oxide for the ALM.

It is well known that the $\mathrm{MoO}_{3}$ melted by laser irradiation in liquid phase exhibits strong acid and oxidant properties [3, 4, building an $\mathrm{Fe}_{2}\left(\mathrm{MoO}_{4}\right)_{3}$ interface layer between the substrate and amorphised $\mathrm{MoO}_{3}$. The coating obtained shows a dark colour due to oxygen vacancies [5], so that no additional pigments for colourising are necessary.

The equipment for the production of a marking coating consists of an industrial laser engraver JQ6090 with a sealed $\mathrm{CO}_{2}$ laser tube with a maximal output power of $80 \mathrm{~W}$.

The industrial application of the presented modification of the ALM requires line-art scanning of the assigned topology, so that the desired image is obtained.

\subsection{ALM PRINTED BARCODE SAMPLE}

Fig. 1 shows a real barcode sample, printed using the suggested ALM, containing the coded sequence of numbers 9788420532318 with a magnified microscopic excerpt from selected bar edges. The picture is obtained by using commercial digital camera Sony HD. A magnified view (objective $4 \times$, ocular $16 \times$ ) of a region, observed by an optical microscope Motic BM13, is situated in the square below the barcode.

\subsection{EXPERIMENTAL SAMPLE}

Using the ALM a sequence of bars of different widths is obtained by irradiating a layer of pre-coated $\mathrm{MoO}_{3}$ powder on stainless steel with the following the topology of a given design (Fig. 2). The working modes (intensity of the laser beam and the scan velocity) are chosen in such a way to make a well bonded black layer on the stainless steel substrate [3, 4].

A sequence of bars consisting of laser lines shifted by $50 \mu \mathrm{m}$ is produced (in Fig. 2 from left to right), The width of the narrowest bar is approximately equal to the diameter of the diffraction spot. Every consecutive

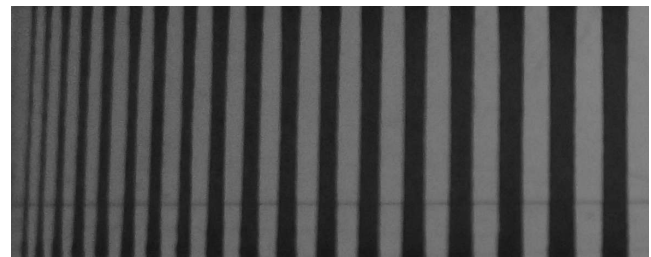

Figure 2. Digital photo of a sequence of black bars and empty spaces with a progressively increasing width.

empty space has the same width as the previous black bar.

\section{Results}

\subsection{Material properties of the COATing}

\subsubsection{AdHESION}

A standard scratch test is performed by a CSEM Revetest (KNMF KIT Karlsruhe, Gernany) scratch tester.

During the scratch test, no critical load for coating failure is registered. Even more, when the maximal load is reached and the indenter scrapes the substrate, traces of the coating in the substrate can still be observed [3]. These results confirm the excellent adhesion of the coating to the substrate.

\subsubsection{REFLECTANCE OF SUBSTRATE AND COATING}

Optical reflectance measurements are performed in the whole visible spectrum by using an AvaSpec spectrophotomer.

Fig. 3 presents the optical reflectance of both the coating and the substrate, which are an important characteristic for the barcode and $2 \mathrm{D}$ code recognition as required by the standard [6].

A large difference between the substrate and coating reflectance is observed. In the spectral region $600-$ $700 \mathrm{~nm}$, in which the most barcode readers operate, the difference is nearly constant (app. 68\%) which meets the GS1 requirements (at least 15\%, [7]).

\subsection{BARCODE RECOGNITION PROPERTIES}

\subsubsection{SCAN REFLECTANCE PROFILE}

To receive the scan reflectance profile, the bar pattern, obtained by the ALM, is illuminated by a light source of a wavelength in the red region, imitating the barcode reader, scanning the bars along a given scan line.

The intensity of each pixel from the bitmap image along the scan path is acquired from a bitmap image of the pattern. The profile is graphically represented in Fig. 4. The regions with high reflectance levels are related to the white spaces; the regions with low levels - to the black bars, as shown in Fig. 4. All barcode symbol parameters, defined in [6, measured experimentally, are listed in the next subsections. 


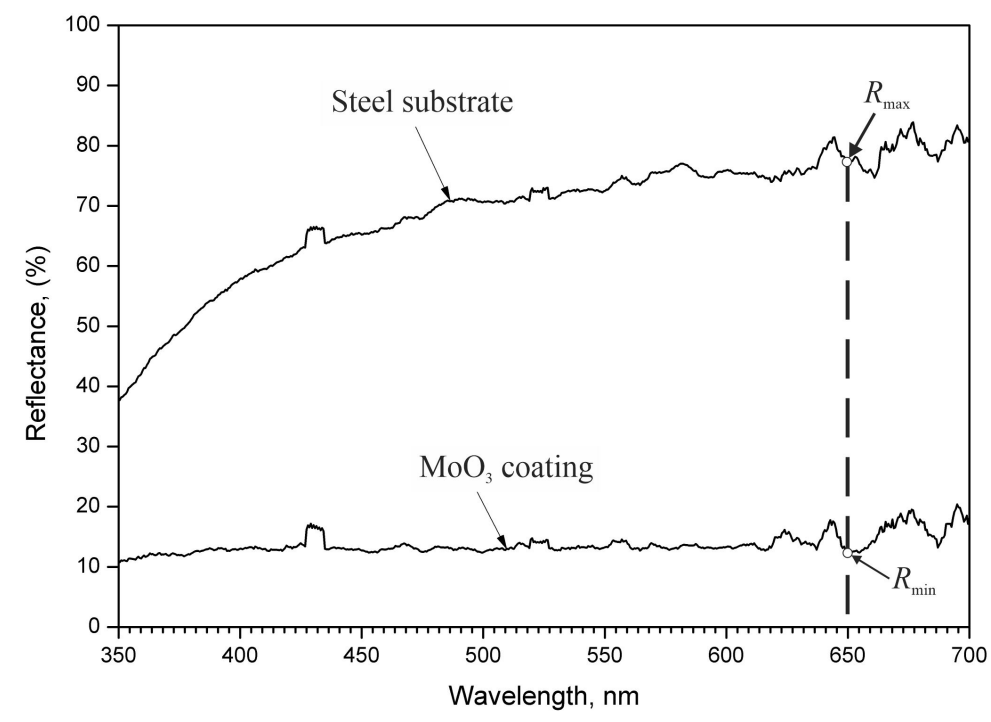

Figure 3. Spectral Reflectance Profile of stainless steel substrate and $\mathrm{MoO}_{3}$ layer, obtained by the ALM.

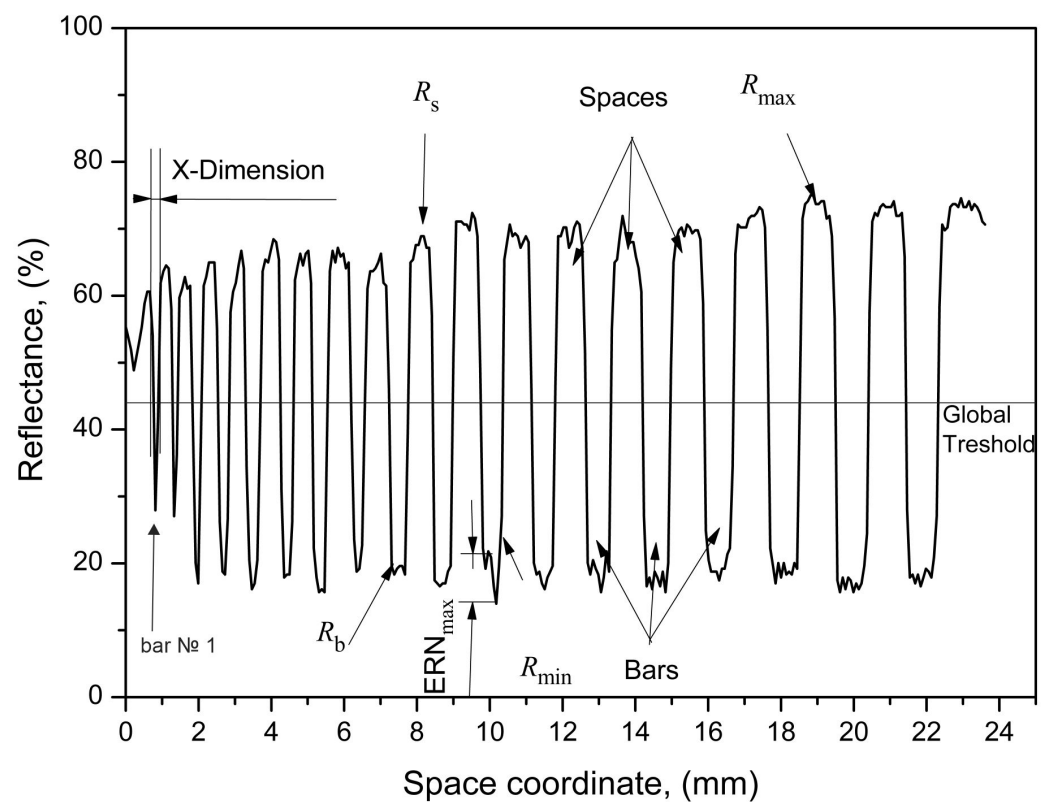

Figure 4. Intensity Profile along a line across the black bars and empty spaces (a. u.). 


\begin{tabular}{ccc}
\hline$R_{\max }$ & $R_{\min }$ & Global Threshold \\
\hline $75 \%$ & $12 \%$ & $44 \%$ \\
\hline
\end{tabular}

TABLE 1. Minimal and maximal reflectance.

\subsubsection{Global Threshold}

In order to distinguish a bar, more precisely, its width, the reflectance level must be under a certain value, defined as Global Threshold. In compliance with [6], the Global Threshold is a horizontal line in the middle between the minimal $R_{\min }$ and maximal $R_{\max }$ level of the pattern reflectance, as shown in Fig. 4 and Table 1 .

\subsubsection{Symbol CONTRAST (SC)}

Generally, the contrast defines the possibility to distinguish between a bar and an empty space. The Symbol Contrast $S C$ is an integral measure for the readability of the barcode. The $S C$ is related to the highest and lowest levels of the reflectance of the reflected light in the bar pattern. Usually, barcode scanners operate at a wavelength of $650 \mathrm{~nm}$, so from Fig. 3, the evaluated values are $R_{\max }=77 \%, R_{\min }=12 \%$ :

$$
S C=R_{\max }-R_{\min }=77 \%-12 \%=65 \%,
$$

which is in a compliance with the standard.

\subsubsection{EdGe CONTRAst (EC)}

Edge contrast is the local reflectance difference between two adjacent empty spaces and a black bar.

Similarly to $S C$, the Edge Contrast is defined as the difference of the highest and lowest reflectance values ( $R_{s}$ and $R_{b}$ respectively) in a pair of adjacent elements (bar + space or space + bar $)$.

From the intensity profile (Fig. 4), $R_{s}$ and $R_{b}$ are evaluated in Table 2 for each bar:

$$
E C=R_{s}-R_{b}
$$

In the worst case, $E C_{\min }=32.7 \%$ is reached.

\subsubsection{Modulation (MOD)}

Modulation is the ratio of the Edge Contrast to the Symbol Contrast:

$$
M O D=E C_{\min } / S C
$$

Modulation is estimated to be $M O D=\frac{32.7 \%}{65 \%}=0.5$.

3.2.6. Defects: $D=E R N_{\max } / S C$

Spots in the empty spaces or light areas in bars will cause a ripple in the scan reflectance profile at the point where the scan path crosses them. This is referred to as Element Reflectance Non-uniformity (ERN, Fig. 2). In the profile of a space, they show as a valley; in that of a bar, they show as a peak. If this peak or valley approaches the threshold between light and dark, the risk of the element being seen as more than one, and of the scan failing to decode, increases.
The defect parameter $D$ is defined as:

$$
D=E R N_{\max } / S C
$$

From Fig. 4 it can be seen, that the maximal $E R N_{\max }$ is most expressed in bar No. 11:

$$
E R N_{\text {max }}=21.9 \%-13.8 \%=8.1 \%
$$

Than the defect parameter is estimated to be:

$$
D=\frac{8.1 \%}{65 \%}=0.13
$$

\subsubsection{MiNIMAL RECOGNIZED BAR WIDTH (X-Dimension)}

In order to recognize the bar code, the minimal width of the bar, more precisely, the space width, must be evaluated (X-Dimension). In an accordance with the [5], this value must be in a range between 264 and $660 \mathrm{\mu m}$.

Technologically, the narrowest possible bar can be obtained by moving the $\mathrm{CO}_{2}$ laser spot along a single line, which is the case with bar No. 1.

From Fig. 4. X-Dimension (XD) is estimated as the space between both dimension lines, entitled " $\mathrm{X}$ Dimension", which defines the well expressed initial and the final points of the bar. From the graph, the $\mathrm{XD}$ is evaluated to be approximately $270 \mu \mathrm{m}$, which is in a compliance with the standard as well as with the diffraction spot size when a lens with a focal length of $50 \mathrm{~mm}$ for the $\mathrm{CO}_{2}$ laser is used as it is in our case [3].

\section{Discussion}

Marking of stainless steel was always a challenge because of its high direct reflectance. Independent of the high reflectance coefficient, the direct reflected light does not reach the reader again, reducing the readability.

Table 3 compares the requirements of the standard and experimental results obtained.

All low levels of reflectance (Fig. 4) are below the global threshold (44\%), even in the case of the narrowest dark bar No. 1 [5, 6].

The minimal width, which can be recognized, is estimated as $270 \mu \mathrm{m}$ - under the required minimal width of $266 \mu \mathrm{m}$.

From Table 3, it can be seen that all parameters of the pattern are in a compliance with the requirements of the standard [6. They are all between Grade 3 and 4 (i. e., the barcode symbol produced by using the ALM is expected to be of a high quality and reliably recognizable by a barcode reader).

Clearly, all parameters can be improved by choosing more suitably selected technological conditions of the ALM process - feed rate, intensity of the laser beam, overlapping distance of the laser scan lines. But, even in the worst case of mirror finish stainless steel with working parameters not perfectly optimised, a high contrast barcode symbol is obtained. 


\begin{tabular}{ccccccccccccccc}
\hline Bar No. & 1 & 2 & 3 & 4 & 5 & 6 & 7 & 8 & 9 & 10 & 11 & 12 & 13 & 14 \\
\hline$E C, \%$ & 32.7 & 37.5 & 46.2 & 47.5 & 51.5 & 58.9 & 49.7 & 48.4 & 52.8 & 55.8 & 56.7 & 54.5 & 58.0 & 59.8 \\
\hline
\end{tabular}

TABLE 2. Edge contrast.

\begin{tabular}{ccccc}
\hline \multicolumn{5}{c}{ Required parameters } \\
\hline Grade & Symbol Contrast & Edge Contrast & Modulation & Defects \\
\hline 4 & $\geq 70 \%$ & $\geq 15 \%$ & $\geq 0.7$ & $\leq 0.15$ \\
3 & $\geq 55 \%$ & & $\geq 0.6$ & $\leq 0.20$ \\
2 & $\geq 40 \%$ & $\geq 0.5$ & $\leq 0.25$ \\
1 & $\geq 20 \%$ & $\geq 0.4$ & $\leq 0.30$ \\
$0($ Fail $)$ & $<20$ & $<15 \%$ & $<0.4$ & $>0.30$ \\
\hline \multicolumn{5}{c}{ Experimental results (worse case) } \\
\hline
\end{tabular}

TABLE 3. Comparison between standard requirements and experimental results.

\section{Conclusions}

- A modification of the ALM method, which needs only one transition metal oxide for a preparation of a high contrast coating over a well-polished stainless steel substrate, chemically-well bonded to the metal, without any additional materials and cleaning substances, has been developed and presented. The coatings show strong adhesion, high hardness, long durability and a high optical contrast.

- The ALM is a suitable technique for direct laser printing of barcodes on stainless steel details, especially made from mirror finished material.

\section{ACKNOWLEDGEMENTS}

The support of KNMF, Karlsruhe Institute of Technology (KIT), Karlsruhe, Germany, Institute of Applied Materials/Applied Materials Science at KIT and German Academic Exchange Service (DAAD) is gratefully appreciated.

\section{REFERENCES}

[1] ISO/IEC 15415 - Information technology - Automatic identification and data capture techniques - Bar code symbol print quality test specification -

Two-dimensional symbols. Standard, International

Organization for Standardization, Geneva, 2011.
[2] NASA-STD-6002D - Applying data matrix identification symbols on aerospace parts. Standard, National Aeronautics and Space Administration, Washington, 2002.

[3] M. S. Mihalev, C. M. Hardalov, C. G. Christov, et al. Transition metal oxides as materials for additive laser marking on stainless steel. Acta Polytechnica 57(4):252 - 262, 2017. DOI:10.14311/AP.2017.57.0252.

[4] M. Mihalev, C. Hardalov, C. Christov, et al. Structural and adhesional properties of thin $\mathrm{MoO} 3$ films prepared by laser coating. Journal of Physics: Conference Series 514, 2014. DOI:10.1088/1742-6596/514/1/012022

[5] N. V. Borisova, E. P. Surovoy. Zakonomernosty izmeneniya opticheskih svoistv nanorazmernyh sloev oxida molibdena (VI) v resultate termoobrabotki. Izvestiya Tomskogo polytechnicheskogo universiteta 310(3):68 - 72, 2007. http://earchive.tpu.ru/handle/11683/1659

[6] ANSI INCITS 182 - for Information Systems: Bar Code Print Quality Guideline. Standard, American National Standards Institute, Washington, 1990.

[7] Bar code verification for linear symbols, ver. 4.3. report, GS1, Washington, 2009. 